\title{
3 Research Square \\ Spread of Endemic SARS-CoV-2 Lineages in Russia Before April 2021
}

Galya V. Klink

A.A. Kharkevich Institute for Information Transmission Problems of the Russian Academy of Sciences

Ksenia R. Safina

Skolkovo Institute of Science and Technology (Skoltech)

Sofya K. Garushyants

A.A. Kharkevich Institute for Information Transmission Problems of the Russian Academy of Sciences

Mikhail Moldovan

Skolkovo Institute of Science and Technology (Skoltech)

Elena Nabieva

Skolkovo Institute of Science and Technology (Skoltech)

Andrey B. Komissarov

Smorodintsev Research Institute of Influenza

Dmitry Lioznov

Smorodintsev Research Institute of Influenza

Georgii A Bazykin ( $\sim$ g.bazykin@skoltech.ru )

Skolkovo Institute of Science and Technology (Skoltech)

\section{Research Article}

Keywords: COVID-19 pandemic, glycoprotein, combinations of mutations

Posted Date: September 1st, 2021

DOI: https://doi.org/10.21203/rs.3.rs-827916/v1

License: (c) (i) This work is licensed under a Creative Commons Attribution 4.0 International License.

Read Full License 


\section{Abstract}

In 2021, the COVID-19 pandemic is characterized by global spread of several lineages with evidence for increased transmissibility. Russia is among the countries with the highest number of confirmed COVID-19 cases, making it a potential hotspot for emergence of novel variants. Here, we show that among the globally significant variants of concern, alpha (B.1.1.7), beta (B.1.351) or gamma (P.1), none have been sampled in Russia before January 2021. Instead, between summer 2020 and spring 2021, the epidemic in Russia has been characterized by the spread of two lineages that are rare elsewhere: B.1.1.317 and a sublineage of B.1.1 including B.1.1.397 (hereafter, B.1.1.397+). Their frequency has increased in different parts of Russia. Mutational composition and frequency dynamics suggest that B.1.1.317 and B.1.1.397+ may be more transmissible than the previously predominant B.1.1. On top of these lineages, in January 2021, B.1.1.7 emerged in Russia, reaching the frequency of 17.4\% (95\% C.I.: 12.0\%-24.4\%) in March 2021. Additionally, we identify three novel distinct lineages, AT.1, B.1.1.524 and B.1.1.525, that have started to spread, together reaching the frequency of $11.8 \%$ (95\% C.I.: 7.5\%-18.1\%) in March 2021. These lineages carry combinations of several notable mutations, including the $S$ :E484K mutation of concern, deletions at a recurrent deletion region of the spike glycoprotein (S: $\Delta 140-142, S: \Delta 144$ or S: $\Delta 136-144$ ), and nsp6: $\Delta 106-$ 108 (also known as ORF1a: $\triangle 3675-3677$ ). Community-based PCR testing indicates that these variants have continued to spread in April 2021, with the frequency of B.1.1.7 reaching 21.7\% (95\% C.I.: 12.3\%-35.6\%), and the joint frequency of B.1.1.524 and B.1.1.525, 15.2\% (95\% C.I.: 7.6\%-28.2\%). Although these variants have been displaced by the onset of delta variant in May-June 2021, the frequency increase of lineages B.1.1.317, B.1.1.397+, AT.1, B.1.1.524 and B.1.1.525 suggest that the combinations of mutations observed in them could have increased the rate of their spread.

\section{Introduction}

Continuing evolution of SARS-CoV-2 in humans leads to emergence of new variants with novel epidemiological and/or antigenic properties. In spring 2020, the S:D614G change has spread globally due to its fitness advantage ${ }^{1,2}$. Subsequently, a number of variants of concern, including alpha (B.1.1.7) first sampled in Great Britain in September, beta (B.1.351) first sampled in South Africa in October, gamma (P.1) first sampled in Brazil in December and delta (B.1.617.2) fist sampled in India in October, were shown to be associated with increased transmissibility ${ }^{3-5}$. These variants are characterized by overlapping sets of changes in spike receptor-binding domain which affect ACE2 binding and antibody recognition, as well as other changes with demonstrated functional and antigenic effects. Emergence of SARS-CoV-2 variants with evidence for change in transmissibility, and possibly other properties, highlights the importance of continued surveillance of novel variants. In particular, locally arising variants that grow in frequency over time may suggest a transmission advantage, although such an increase may also occur by chance ${ }^{6}$.

Here, we show that before spring 2021, the outbreak in Russia was characterized by a spread of two lineages, B.1.1.317 and B.1.1.397+, which were highly prevalent in Russia but rarely appeared in nonRussian samples. We trace the accumulation of sequential mutations in the evolution of these lineages, 
and single out the spike mutations that were followed by bursts in frequency. If the frequency increase of B.1.1.317 and B.1.1.397 + has been indeed driven by changes in the intrinsic properties of the virus rather than by epidemiology, it is these mutations in spike that most likely have led to this increase, although importantly we lack direct transmission data to verify causality. We also describe three novel candidate variants of interest that are characterized by a rapid increase in frequency and combinations of important spike mutations, including the E484K mutation of concern.

\section{Results}

High-frequency variants in Russia

We analyzed 4,487 SARS-CoV-2 sequences with known sampling dates obtained in Russia between February 25, 2020 - March 28, 2021. 2,842 of these samples are deposited to GISAID ${ }^{7}$, while the remaining 1,645 (all dating to February-November 2020) will be made available through another repository. All 968 analyzed samples from December 2020 - March 2021 are available on GISAID. The vast majority of samples over this period came from several genomic surveillance programs which were not targeted towards particular variants, although representation of Russia's regions varied with time.

Throughout the pandemic, the SARS-CoV-2 diversity in Russia has been predominated by the B.1.1 Pango lineage which is frequent in Europe, as well as lineages descendant from it ${ }^{8}$ (Fig. 1). Three B.1.1-derived lineages with the highest prevalence in Russia in the beginning of 2021 were B.1.1.7, which was firstly introduced in Russia at the end of 2020, as well as two other lineages, B.1.1.317 and B.1.1.397, that appeared in Russia in April and July, 2020, respectively. B.1.1.317 was first sampled in Vietnam on March 27, 20209; within Russia, it was first sampled on April 5, 2020 in Moscow, spreading across the country throughout 2020 (Fig. 2). B.1.1.397 was first sampled in the Krasnoyarsk Region of Russia on July 22, 2020. By summer 2020, both B.1.1.317 and B.1.1.397 were frequent throughout Russia (Fig. 2).

To find the non-reference amino acid variants that gained in frequency in Russia, we selected the positions at which the mean frequency of the non-reference variant in Russian samples exceeded $5 \%$ (for the spike) or 10\% (for other proteins) in February-March 2021. We found 21 such positions in spike and 21 such positions in other proteins. Among these changes, two (RdRp:P323L and S:D614G) were fixed early in the global evolution of SARS-CoV-2; other two (N:R203K and N:G204R) are the lineage-defining mutations of B.1.1.

The frequency dynamics of the derived variants at the remaining 38 positions is shown in Fig. 3 . These include the mutations characterizing the B.1.1.7 variant which has been increasing in frequency in Russia since January 2021 (Fig. 1), as well as some of the other globally spreading mutations of concern or interest, including the E484K mutation in spike. However, at many of these sites, the non-reference variants were rare outside Russia (Fig. 3). Most of these variants showed similar temporal dynamics in Moscow and St. Petersburg regions, as well as in the European and Asian parts of Russia (Fig. S1), indicating that their increase in frequency is not a result of sampling bias. 
We aimed to identify the high-frequency variants carrying these mutations. Many of these sites were highly homoplasic, and overall we found the resulting phylogenies not to be robust. Instead, we defined the most frequent variants composed of these mutations, independent of the alleles at other sites (Fig. 4).

We considered the allele combinations that were most frequent in Russia in February-March 2021, and noticed that they belonged to several nested sets. The most frequent combination ( 99 out of the 461 samples) carried the N:A211V mutation which is characteristic of the B.1.1.317 Pango lineage; the second most frequent combination carried the S:D138Y and ORF8:V62L combination of mutations which are characteristic of the B.1.1.397 lineage; the third combination carried the set of characteristic mutations of B.1.1.7.

Still, there was no one-to-one correspondence between the frequent combinations of mutations and Pango lineages. For example, while the most frequent variant carrying S:M153T was that also including N:M234I, S:D138Y and ORF8:V62L (column 2 in Fig. 4), and classified as Pango lineage B.1.1.397, the variants carrying S:M153T alone, the S:M153T + N:M234I combination and the S:M153T + N:M234I + S:N679K combination were also frequent (columns 4, 9 and 5 in Fig. 4 respectively) but were classified by PANGOLIN as other lineages (B.1.1, B.1.1.141, B.1.1.28 and others). Similarly, while B.1.1.317 is defined by the N:A211V mutation, the frequency of the variant carrying this mutation alone was relatively low (column 12 in Fig. 4), while most samples carrying it also carried 8 additional high-frequency mutations, including four potentially important changes in spike (Q675R + D138Y + S477N + A845S; column 1 in Fig. 4). The frequencies of such "non-canonical" combinations of mutations increased throughout 20202021 (Fig. 5).

Finally, we observe three high-frequency combinations of mutations, including the S:E484K mutation of concern as well as other mutations of interest (notably $S: \Delta 140-142, S: \Delta 136-144$ and nsp $6: \Delta 106-108$, also referred to as ORF1a: $\Delta 3675-3677$; columns 7, 8 and 10 in Fig. 4). These combinations have recently got B.1.1.524, AT.1 and B.1.1.525 Pango designations (for columns 7, 8 and 10 in Fig. 4, respectively).

Frequency dynamics of the variants prevalent in Russia

To study potential effects of individual mutations composing a variant on the frequency dynamics of this variant, we fit the logistic growth model for the 10 most-frequent combinations of mutations and for $\mathrm{N}: \mathrm{A} 211 \mathrm{~V}$ (which was the 12th most-frequent combination), and compared the dynamics of nested combinations with each other (Figs. 6-8).

The variant carrying just the N:A211V change (largely coincident with the B.1.1.317 Pango lineage) has increased in frequency since the start of the epidemic in Russia. However, since fall 2020, it was being displaced by the variant with 8 additional mutations, including four in spike: Q675R + D138Y + S477N + A845S. When the logistic growth model was fit to the N:A211V variant alone, it demonstrated modest growth (Fig. 6A); however, its combination with S:Q675R + D138Y + S477N + A845S demonstrated a much more rapid frequency increase, with the estimated daily growth rate of $1.93 \%$ (95\% Cl: $1.8 \%-2.06 \%$; Fig. 6B). While this led to a frequency increase of the N:A211V mutation independent of the background 
(Fig. 6C), this suggests that the frequency increase was more likely driven by the S:Q675R + D138Y + S477N + A845S combination than the N:A211V change defining the B.1.1.317 Pango lineage.

By contrast, the frequency of the S:M153T mutation grew independently of the presence of other mutations from our list (Fig. 7). While subsequent mutations may add to the estimated growth rates, these rates were comparable when the S:M153T mutation occurred alone or in combination with $\mathrm{N}: \mathrm{M} 234 \mathrm{I}, \mathrm{N}: \mathrm{M} 234 \mathrm{I}+\mathrm{S}: \mathrm{N} 679 \mathrm{~K}$, or N:M234I + S:D138Y, and all these combinations were still frequent many months after they had originated (Fig. 5, 7).

Finally, the five remaining variants which also reached high frequency in February-March carried unnested, although partially overlapping sets of mutations. These included B.1.1.7 (Fig. 8A); a variant carrying the S:P681H mutation of interest in the absence of other high-frequency mutations (Fig. 8B); as well as three novel variants carrying the following combinations of mutations: (i) nsp6: $\Delta 106-108+$ S:P9L + S: $\triangle 140-142$ (which recently got the B.1.1.524 Pango designation; Fig. 8C); (ii) S:P9L + S: $\triangle 136-$ $144+$ S:E484K (which recently got the AT.1 Pango designation; Fig. 8D); and (iii) nsp6: $\Delta 106-108+$ S: $\triangle 144+$ S:E484K (which recently got the B.1.1.525 Pango designation; Fig. 8E). Variants B.1.1.524, AT.1 and B.1.1.525 were only observed in 13-14 samples each, but are of interest because this constitutes an appreciable fraction of samples obtained in February-March (3.0\%, 2.8\% and $2.6 \%$ respectively), and also because they are composed of known mutations of interest or concern. The daily growth rate estimated for these variants by the logistic growth model was in the range of $2.44-7.18 \%$ (Fig. 8C-E).

The continued spread of some of these variants between February-April 2021 was confirmed by community-based PCR testing. To obtain independent frequency estimates, we made use of a PCR system sensitive to the presence of nsp6: $\Delta 106-108$ and $S: \Delta 69-70$ deletions (see Methods) to detect the B.1.1.7, B.1.1.524 and B.1.1.525 variants. Specifically, S: $\Delta 69-70^{+} n s p 6: \Delta 106-108^{+}$samples correspond to B.1.1.7, while S: $\Delta 69-70^{-}$nsp6: $\Delta 106-108^{+}$samples correspond to either the B.1.1.524 or the B.1.1.525 variant (Fig. 4). While the frequency estimates were highly uncertain (Table 1), they indicate that B.1.1.7, and one or both of variants B.1.1.524 and B.1.1.525, were wide-spread in April (Fig. 9, Table 1). A considerable fraction (59.6\%) of PCR samples from February and March were included in our main analysis, as their sequences were in GISAID. However, the frequency increase was also observed in the 136 PCR samples for which no sequencing data was available (Fig. S2), providing independent validation of the NGS results. Similarly, it was observed when the PCR tests only for St. Petersburg were analyzed (Fig. S3, S4), indicating that the prevalence of these variants increased at least in this city as opposed to being an artefact of changing sampling between regions. 
Table 1

Frequencies of (B.1.1.524 or B.1.1.525) and B.1.1.7 estimated from PCR data. The point estimate and the $95 \%$ confidence intervals (Wilson score intervals) are shown.

\begin{tabular}{|c|c|c|c|c|}
\hline & \multicolumn{2}{|c|}{ 2021-Feb } & 2021-Mar & 2021-Apr \\
\hline \multirow{2}{*}{$\begin{array}{l}\text { nsp6: } \Delta 106-108+S: \Delta 69-70-(B .1 .1 .524 \text { or } \\
\text { B.1.1.525) }\end{array}$} & \multicolumn{2}{|l|}{$10.5 \%$} & $6.9 \%$ & $15.2 \%$ \\
\hline & \multicolumn{2}{|c|}{$(2.3 \%-31.4 \%)$} & $(4.1 \%-11.2 \%)$ & $(7.6 \%-28.2 \%)$ \\
\hline \multirow[t]{2}{*}{ nsp6: $\Delta 106-108+S: \Delta 69-70+(B \cdot 1.1 .7)$} & \multicolumn{2}{|l|}{$10.5 \%$} & $13.7 \%$ & $21.7 \%$ \\
\hline & \multicolumn{2}{|c|}{$(2.9 \%-31.4 \%)$} & $(9.7 \%-19.1 \%)$ & $(12.3 \%-35.6 \%)$ \\
\hline & 2021-Feb & \multicolumn{2}{|c|}{ 2021-Mar } & 2021-Apr \\
\hline $\begin{array}{l}\text { nsp6: } \Delta 106-108+\mathrm{S}: \Delta 69-70-(B \cdot 1.1 .524 \text { or } \\
\text { B.1.1.525) }\end{array}$ & $\begin{array}{l}10.5 \% \\
(2.3 \%-31.4 \%)\end{array}$ & \multicolumn{2}{|c|}{$\begin{array}{l}6.9 \% \\
(4.1 \%-11.2 \%)\end{array}$} & $\begin{array}{l}15.2 \% \\
(7.6 \%-28.2 \%)\end{array}$ \\
\hline nsp6: $\Delta 106-108+S: \Delta 69-70+(B \cdot 1.1 .7)$ & $\begin{array}{l}10.5 \% \\
(2.9 \%-31.4 \%)\end{array}$ & \multicolumn{2}{|c|}{$\begin{array}{l}13.7 \% \\
(9.7 \%-19.1 \%)\end{array}$} & $\begin{array}{l}21.7 \% \\
(12.3 \%-35.6 \%)\end{array}$ \\
\hline
\end{tabular}

Mutational composition of the high-frequency variants

In this section, we discuss the mutations that constitute the variants that were spreading in Russia before April 2021.

B.1.1.317. This lineage is defined by the presence of the N:A211V mutation. Changes at nucleocapsid position 211 experienced both persistent (according to the FEL model of HyPhy ${ }^{10}$ ) and episodic (according to the MEME model ${ }^{11}$ ) positive selection both in the global ${ }^{12}$ and in the Russian dataset $(p=$ 0.0396 for the MEME model and $p=0.0268$ for the FEL model, the likelihood-ratio test), as well as a rapid increase in frequency of non-reference variants in the global dataset ${ }^{12}$. While the global frequency of $211 \mathrm{~V}$ has remained low $(<0.4 \%)$, in Russia, it has reached 26.9\% in February-March 2021. According to immunoinformatic analysis, site $\mathrm{N}: 211$ is included in one of the four regions of the nucleocapsid protein with the highest affinity to multiple MHC-I alleles ${ }^{13}$. Nevertheless, the frequency of the variant carrying the $\mathrm{N}$ :A211V mutation alone has declined since October 2020 (Fig. 5), suggesting that it is unlikely to confer transmission advantage against the background of other variants that were frequent in early 2021 (Fig. 6).

A subclade within B.1.1.317 that was spreading rapidly during the studied period carried the (Q675R+ $\mathrm{D} 138 \mathrm{Y}+\mathrm{S} 477 \mathrm{~N}+\mathrm{A} 845 \mathrm{~S}$ ) combination of changes in spike. Two of these mutations are of interest. $\mathrm{S}: \mathrm{D} 138 \mathrm{Y}$, first described as one of the lineage-defining mutations of the P.1 lineage ${ }^{14}$, is a change in the $\mathrm{N}$ terminal domain (NTD) of spike. Site 138 is adjacent to the NTD antigenic supersite, and together with other NTD mutations of P.1, S:D138Y was suggested to be the cause of disruption of binding with mAb159 ${ }^{15}$ which is one of the most potent inhibitory antibodies ${ }^{16}$. S:S477N is positioned in the receptorbinding motif (RBM) of the S-protein near the antibody binding site (Fig. 10) and was reported to promote resistance to multiple antibodies and plasma from convalescent patients ${ }^{17,18}$. Additionally, $S 477 \mathrm{~N}$ is 
thought to increase ACE2 binding ${ }^{19}$. It is one of the lineage-defining mutations of the B.1.160 (20A.EU2) variant of interest prevalent in Europe in Autumn $2020^{20}$, and the only one among them to occur in the Sprotein; it also defines one of the two subclades of the B.1.526 variant of interest that were spreading in the USA in the beginning of $2021^{21,22}$. S:Q675R is located at the central part of S1 (Fig. 10), and S:A845S, in S2; the significance of these two mutations is unknown.

B.1.1.397+. S:M153T is a characteristic mutation of B.1.1.397, which also comprises several other mutations. However, the frequency of S:M153T in Russia also increased in the absence of these other mutations (Fig. 7). This increase has been ongoing since late spring 2020 (Fig. 5), and has been noticed in Russia ${ }^{23,24}$. S:M153T, however, has remained rare outside Russia. S:153 is the first position of the 6amino acid insertion specific to SARS-CoV-2 and some closely related bat betacoronaviruses that was absent in SARS-CoV25. While the effect of S:M153T on antigenic properties is unknown, S:153 is a part of the N3 loop of the NTD. This loop is a part of the NTD antigenic supersite (Fig. 10; ${ }^{26}$ ), and nearby residues, including S:152, were recently shown to bind highly neutralizing 4A8 antibody from a convalescent patient ${ }^{27}$. Besides Russia, S:M153T was prevalent in Kazakhstan ${ }^{28}$ which has a long border with Russia, suggesting common ancestry of this change in these countries.

The most frequent subclade within B.1.1.397+, and the one with some evidence for an independent increase in frequency (Fig. 7), was defined by the presence of two additional mutations of interest: S:D138Y discussed above in the context of the B.1.1.317 lineage (but acquired in the B.1.1.397 lineage independently); and N:M234l. Position N:234 is a part of a disordered linker domain of the nucleocapsid protein $^{29}$. Outside B.1.1.397, the N:M234I change has also occurred independently in several lineages that attracted attention. It is among the lineage-defining mutations of the B.1.160 (20A.EU2) lineage as well as the B.1.526 lineage that increased in frequency in the USA at rates comparable to those of B.1.1.721. It was also one of the changes defining a lineage (preliminarily identified as B.1.x) which also contained S:N501Y and S:P681H and seemed to spread rapidly in the USA ${ }^{30}$. Independent emergence of $\mathrm{N}: \mathrm{M} 234 \mathrm{I}$ in several variants of interest may reflect its impact on at least one of multiple functions of the $\mathrm{N}$ $\operatorname{protein}^{31}$.

Other notable variants. The five other combinations of mutations observed at high frequencies in Russia in February-March 2021 were B.1.1.7, the best-known variant with increased transmissibility before the emergence of B.1.617.2 lineage; the variant carrying the S:P681H mutation alone; and three novel variants.

$\mathrm{S}: \mathrm{P} 681 \mathrm{H}$ is one of the nine spike changes that characterized B.1.1.7 lineage ${ }^{3}$; and S:P681R is one of lineage-defining mutations of B.1.617.2 lineage; however, changes in this site are absent from the two other lineages of concern, B.1.351 and P.1, indicating that it is not essential for increased transmissibility. The 681 position is adjacent to the furin cleavage site; this site is absent in non-human CoV, and is assumed to have contributed to pathogenicity in humans ${ }^{32}$. Changes at this position experienced both persistent and episodic positive selection ${ }^{12}$. $\mathrm{P} 681 \mathrm{H}$ appeared to increase in frequency globally ${ }^{33}$, 
although it was hard to disentangle this increase from that of the other changes constituting B.1.1.7 lineage that was rapidly spreading. We find that the frequency of this mutation in Russia in the absence of other B.1.1.7 mutations did not increase (Fig. 8), indicating that it does not increase transmissibility by itself.

The three remaining high-frequency variants with evidence for rapid frequency increase in early 2021 carried combinations of the following high-frequency mutations: S:P9L, S: $140-142$ (or S: $\Delta 136-144$ ), S:E484K, and nsp6: $\Delta 106-108$. The sets of mutations in these variants are in conflict (i.e., not nested within each other; Fig. 4), indicating that at least some of these mutations emerged in them independently. These mutations are of interest or concern. Specifically, S:E484K (present in AT.1 and B.1.1.525) is involved in several famous variants including the B. $1.351^{4}, P .1^{5,34}$ and P. $2^{5,34}$ lineages, and has been shown by several groups to cause escape from neutralizing antibodies ${ }^{35-37}$. nsp6: $\Delta 106-108$ (also referred to as ORF1a: $\triangle 3675-3677$, and present in B.1.1.524 and B.1.1.525) is a part of three variants of concern (B.1.1.7, B.1.351, P.1). S: $\Delta 140-142$ (present in B.1.1.524), S: $\Delta 144$ (present in B.1.1.525) and $S: \Delta 136-144$ (present in AT.1) are distinct deletions at a recurrent deletion region of the spike glycoprotein which confer resistance to neutralizing antibodies ${ }^{38}$.

\section{Discussion}

Russia has been relatively well isolated in the course of COVID-19 pandemic: both the first cases of COVID-19 and the arrival of variants of concern, notably the B.1.1.7, have happened here later than in many European countries ${ }^{8,39}$. Together with the large size of the outbreak in Russia, such isolation could have created conditions for emergence of novel important domestic variants.

A steady increase in frequency of lineages B.1.1.317 and B.1.1.397+, as well as the presence of multiple mutations with potential effect on antigenic properties, notably S:D138Y, merit classification of these two lineages as variants of interest ${ }^{40,41}$. Nevertheless, the rate of spread of B.1.1.317 and B.1.1.397 + has been lower than that of VOCs (e.g., 7\% for B.1.1.742). In particular, while B.1.1.317 has been observed in Russia since April 2020, the subclade of B.1.1.317 carrying the three spike mutations, since July 2020, and B.1.1.397+, since April 2020, all these lineages have remained at frequencies below $30 \%$ in Russia, and the logistic growth rates estimated by our model are all below $2 \%$ (Fig. 6-8). Besides, these variants were missing spike changes L452R, E484K or N501Y which occur in other VOCs ${ }^{41}$. All these variants have been displaced with the advent of the delta variant in summer 2021.

The combinations of mutations seen in the three variants that emerged in 2021, AT.1, B.1.1.524 and B.1.1.525 (Fig. 8C-E), look more suspicious, because their estimated rate of frequency increase was higher and because they include mutations with known effects and occurring in other variants of interest or concern. While these variants have also become rare after the spread of the delta variant, the match 
between their mutational composition and that of other globally spreading variants of concern indicates that the mutations comprising them could have contributed to their rapid frequency increase.

\section{Methods}

Dataset preparation. 1,060,545 sequences of SARS-CoV-2 were downloaded from GISAID on April 15, 2021 (Supplementary File 2) and aligned with MAFFT ${ }^{43}$ v7.45324 against the reference genome WuhanHu-1/2019 (NCBI ID: MN908947.3) with --addfragments --keeplength options. 100 nucleotides from the beginning and from the end of the alignment were trimmed. After that, we excluded sequences (1) shorter than $29,000 \mathrm{bp}$, (2) with more than 3,000 (for Russian sequences) or 300 (for all other countries) positions of missing data (Ns), (3) excluded by Nextstrain ${ }^{44}$, (4) in non-human animals, (5) with a genetic distance to the reference genome more than four standard deviations from the epi-week mean genetic distance to the reference, or (6) with incomplete collection dates. As our focus was on the spread of lineages in Russia, and since Russia is relatively poorly sampled, we chose a less stringent threshold at step (2) for Russian compared to non-Russian sequences in order to keep more Russian data in the dataset. To this dataset, we added the 1,645 Russian sequences described in our earlier study ${ }^{45}$ and 344 samples produced by the CoRGI consortium which had not been yet available in GISAID on April 15 (but have been deposited to GISAID since then). The final dataset consisting of 830,249 sequences, including 4,487 Russian samples, was then annotated by the PANGOLIN package (v2.3.8). For the categories in Fig. 1, we selected Pango lineages represented by at least 100 Russian samples (there were six such lineages: B.1, B.1.1, B.1.1.397, B.1.1.28, B.1.1.317 and B.1.1.294), added the two earliest lineages A and B and the VOC lineage B.1.1.7, and aggregated A, B, B.1 and B.1.1 with lineages nested in them except those included in other categories into A.*, B.*, B.1.* and B.1.1.*, respectively.

Data analysis. To trace the frequency dynamics of mutations, for each non-reference nucleotide at each position in each month, we calculated the fraction of samples with this nucleotide among all samples where this position contained an ambiguous nucleotide. We did this separately for the Russian and nonRussian samples, and selected changes with frequency more than $5 \%$ in $S$ protein and more than $10 \%$ in other proteins in February-March 2021 (a total of 461 sequences) in Russian samples for consideration. Calculations were performed with custom Perl and R scripts. Wilson score intervals were estimated using Hmisc R package ${ }^{46}$, and results were visualized with the ggplot2 package ${ }^{47}$ of $\mathrm{R}$ language ${ }^{48}$. Upset plots were built with the UpSetR package ${ }^{49}$ for R. Logistic growth models were fit to frequencies of each variant averaged across 14-days sliding windows with $\mathrm{nls}\left(\right.$ ) function of R language ${ }^{48}$. Only windows with total number of samples $>20$ were taken into account. Confidence intervals for estimated parameters were obtained with confint2 function from nlstools R package ${ }^{50}$, similar to ${ }^{42}$. Results were visualized with $\mathrm{R}$ packages ggplot $2^{47}$, tidyverse ${ }^{51}$ and gridExtra ${ }^{52}$.

To estimate positive selection, we employed MEME and FEL models implemented in the HyPhy package $^{10,11}$. For this analysis, we added Russian sequences with incomplete collection dates to the main dataset, which resulted in 5006 Russian sequences. The tree for the selection analysis was built 
upon the whole-genome alignment of Russian sequences with the RAxML package v.8.0.26 (model GTRCAT $)^{53}$.

Mapping residues onto the S-protein structure. To visualize spike mutations, we utilized two different spike protein structures, corresponding to S-protein in complex bound with 4A8 (PDB ID: 7c2I) or with P5A-1B9 (PDB ID: 7czx) antibody. The NTD antibody binding epitope is defined as $\mathrm{in}^{26}$. The two structures were structurally aligned and visualized with Open-Source PyMOL ${ }^{54}$.

PCR data. Community-based PCR tests aimed at detection of S: $\Delta 69-70$ and nsp6: $\Delta 106-108$ deletions were performed for 739 samples. We further analyzed only those samples for which both tests were performed and produced unambiguous results. There were 269 such samples from 22 regions (including 170 from Saint Petersburg, 43 from Sverdlovsk Oblast, and 12 from Leningrad Oblast) obtained between February-April, 2021. For S: $\triangle 69-70$ detection ${ }^{55}$, we used the Yale69/70del RT-PCR assay described elsewhere. For nsp6: $\triangle 106-108$ detection, we used a newly designed RT-PCR assay. 133 of these 269 samples were also sequenced (sequence data made available through GISAID); for 126 of them (94.7\%), the results on the presence of $S: \Delta 69-70$ and nsp6: $\triangle 106-108$ were consistent between the NGS and PCR data, indicating that our PCR tests are highly specific.

\section{Declarations}

Acknowledgements

We are grateful to all GISAID submitting and originating labs (Supplementary File 1) for rapid open release of SARS-CoV-2 sequencing data. We thank Sergei L Kosakovsky Pond for help with HyPhy analyses, and Evgeniya Alekseeva and members of the Bazykin lab for fruitful discussions. This work was supported by the RFBR grant 20-54-80014 to G.A.B.

Author contributions

Conceptualization: GVK KRS GAB

Investigation: GVK KRS SKG MM EN ABK DL CORGI

Data Curation: ABK DL CORGI

Formal analysis: GVK KRS MM GAB

Visualization: GVK KRS SKG

Supervision: GAB

Writing-original draft: GVK GAB

Writing-review \& editing: GVK KRS EN GAB 


\section{References}

1. Korber, B. et al. Tracking Changes in SARS-CoV-2 Spike: Evidence that D614G Increases Infectivity of the COVID-19 Virus. Cell 182, 812-827.e19 (2020).

2. Volz, E. et al. Evaluating the Effects of SARS-CoV-2 Spike Mutation D614G on Transmissibility and Pathogenicity. Cell 184, 64-75.e11 (2021).

3. ECDPC. Rapid increase of a SARS-CoV-2 variant with multiple spike protein mutations observed in the United Kingdom. (2020).

4. Tegally, H. et al. Emergence and rapid spread of a new severe acute respiratory syndrome-related coronavirus 2 (SARS-CoV-2) lineage with multiple spike mutations in South Africa. medRxiv 2020.12.21.20248640 (2020) doi:10.1101/2020.12.21.20248640.

5. Genomic characterisation of an emergent SARS-CoV-2 lineage in Manaus: preliminary findings. Virological https://virological.org/t/genomic-characterisation-of-an-emergent-sars-cov-2-lineage-inmanaus-preliminary-findings/586 (2021).

6. Hodcroft, E. B. et al. Emergence and spread of a SARS-CoV-2 variant through Europe in the summer of 2020. medRxiv 2020.10.25.20219063 (2021) doi:10.1101/2020.10.25.20219063.

7. Eurosurveillance I GISAID: Global initiative on sharing all influenza data - from vision to reality. https://www.eurosurveillance.org/content/10.2807/1560-7917.ES.2017.22.13.30494.

8. Komissarov, A. B. et al. Genomic epidemiology of the early stages of the SARS-CoV-2 outbreak in Russia. Nat. Commun. 12, 649 (2021).

9. CoVizu. Near real-time visualization of SARS-CoV-2 (hCoV-19) genomic variation https://filogeneti.ca/CoVizu/ (2021).

10. Kosakovsky Pond, S. L. \& Frost, S. D. W. Not So Different After All: A Comparison of Methods for Detecting Amino Acid Sites Under Selection. Mol. Biol. Evol. 22, 1208-1222 (2005).

11. Murrell, B. et al. Detecting Individual Sites Subject to Episodic Diversifying Selection. PLOS Genet. 8, e1002764 (2012).

12. Selection history of genes in SARS-CoV-2/COVID-19 genomes enabled by data from. https://observablehq.com/@spond/sc2-genes (2021).

13. Oliveira, S. C., de Magalhães, M. T. Q. \& Homan, E. J. Immunoinformatic Analysis of SARS-CoV-2 Nucleocapsid Protein and Identification of COVID-19 Vaccine Targets. Front. Immunol. 11, (2020).

14. Genomics and epidemiology of the P.1 SARS-CoV-2 lineage in Manaus, Brazil | Science. https://science.sciencemag.org/content/early/2021/04/13/science.abh2644.

15. Dejnirattisai, W. et al. Antibody evasion by the P.1 strain of SARS-CoV-2. Cell (2021) doi:10.1016/j.cell.2021.03.055.

16. Dejnirattisai, W. et al. The antigenic anatomy of SARS-CoV-2 receptor binding domain. Cell 184, 2183-2200.e22 (2021). 
17. Gaebler, C. et al. Evolution of Antibody Immunity to SARS-CoV-2. bioRxiv 2020.11.03.367391 (2021) doi:10.1101/2020.11.03.367391.

18. Liu, Z. et al. Landscape analysis of escape variants identifies SARS-CoV-2 spike mutations that attenuate monoclonal and serum antibody neutralization. bioRxiv 2020.11.06.372037 (2021) doi:10.1101/2020.11.06.372037.

19. Serine 477 plays a crucial role in the interaction of the SARS-CoV-2 spike protein with the human receptor ACE2 | Scientific Reports. https://www.nature.com/articles/s41598-021-83761-5.

20. CoVariants. https://covariants.org/variants/20A.EU2.

21. A Novel and Expanding SARS-CoV-2 Variant, B.1.526, Identified in New York | medRxiv. https://www.medrxiv.org/content/10.1101/2021.02.23.21252259v2.full.

22. West, A. P. et al. Detection and characterization of the SARS-CoV-2 lineage B.1.526 in New York. bioRxiv 2021.02.14.431043 (2021) doi:10.1101/2021.02.14.431043.

23. The expert reported on the mutation of the coronavirus in 13 regions of the Russian Federation (in Russian). https://www.interfax.ru/russia/737621.

24. Dangerous COVID-19 mutations, which Popova warned about, were found in the Urals (in Russian). vesti.ru https://www.vesti.ru/article/2486564.

25. Guruprasad, L. Evolutionary relationships and sequence-structure determinants in human SARS coronavirus-2 spike proteins for host receptor recognition. Proteins Struct. Funct. Bioinforma. 88, 1387-1393 (2020).

26. Cerutti, G. et al. Potent SARS-CoV-2 Neutralizing Antibodies Directed Against Spike N-Terminal Domain Target a Single Supersite. bioRxiv 2021.01.10.426120 (2021) doi:10.1101/2021.01.10.426120.

27. Chi, X. et al. A neutralizing human antibody binds to the N-terminal domain of the Spike protein of SARS-CoV-2. Science 369, 650-655 (2020).

28. outbreak.info. outbreak.info https://outbreak.info/.

29. Cubuk, J. et al. The SARS-CoV-2 nucleocapsid protein is dynamic, disordered, and phase separates with RNA. Nat. Commun. 12, 1936 (2021).

30. Thornlow, B. et al. A new SARS-CoV-2 lineage that shares mutations with known Variants of Concern is rejected by automated sequence repository quality control. bioRxiv 2021.04.05.438352 (2021) doi:10.1101/2021.04.05.438352.

31. Gao, T. et al. Identification and functional analysis of the SARS-COV-2 nucleocapsid protein. BMC Microbiol. 21, 58 (2021).

32. The sequence at Spike S1/S2 site enables cleavage by furin and phospho-regulation in SARS-CoV2 but not in SARS-CoV1 or MERS-CoV I Scientific Reports. https://www.nature.com/articles/s41598020-74101-0.

33. Maison, D. P., Ching, L. L., Shikuma, C. M. \& Nerurkar, V. R. Genetic Characteristics and Phylogeny of 969-bp S Gene Sequence of SARS-CoV-2 from Hawaii Reveals the Worldwide Emerging P681H 
Mutation. bioRxiv 2021.01.06.425497 (2021) doi:10.1101/2021.01.06.425497.

34. Voloch, C. M. et al. Genomic characterization of a novel SARS-CoV-2 lineage from Rio de Janeiro, Brazil. medRxiv 2020.12.23.20248598 (2020) doi:10.1101/2020.12.23.20248598.

35. Identification of SARS-CoV-2 spike mutations that attenuate monoclonal and serum antibody neutralization: Cell Host \& Microbe. https://www.cell.com/cell-host-microbe/fulltext/S19313128(21)00044-5.

36. Andreano, E. et al. SARS-CoV-2 escape in vitro from a highly neutralizing COVID-19 convalescent plasma. bioRxiv 2020.12.28.424451 (2020) doi:10.1101/2020.12.28.424451.

37. Greaney, A. J. et al. Comprehensive mapping of mutations in the SARS-CoV-2 receptor-binding domain that affect recognition by polyclonal human plasma antibodies. Cell Host Microbe 0, (2021).

38. McCarthy, K. R. et al. Recurrent deletions in the SARS-CoV-2 spike glycoprotein drive antibody escape. Science 371, 1139-1142 (2021).

39. The first case of the 'British variant' of the coronavirus is detected in Russia (in Russian). Kommersant https://www.kommersant.ru/doc/4639704 (2021).

40. Emma Griffiths et al. CanCOGeN Interim Recommendations for Naming, Identifying, and Reporting SARS-CoV-2 Variants of Concern.

41. CDC. SARS-CoV-2 Variant Classifications and Definitions. Centers for Disease Control and Prevention https://www.cdc.gov/coronavirus/2019-ncov/cases-updates/variant-surveillance/variant-info.html (2021).

42. Washington, N. L. et al. Genomic epidemiology identifies emergence and rapid transmission of SARS-CoV-2 B.1.1.7 in the United States. medRxiv 2021.02.06.21251159 (2021) doi:10.1101/2021.02.06.21251159.

43. Katoh, K. \& Standley, D. M. MAFFT multiple sequence alignment software version 7: improvements in performance and usability. Mol. Biol. Evol. 30, 772-780 (2013).

44. https://github.com/nextstrain/ncov/blob/master/defaults/exclude.txt. GitHub https://github.com/nextstrain/ncov/blob/master/defaults/exclude.txt.

45. Matsvay, A. et al. Genomic epidemiology of SARS-CoV-2 in Russia reveals recurring cross-border transmission throughout 2020. medRxiv 2021.03.31.21254115 (2021) doi:10.1101/2021.03.31.21254115.

46. Harrel, F. E., Jr. \& others, with contributions from C. D. and many. Hmisc: Harrell Miscellaneous. (2021).

47. Wickham, H. ggplot2: Elegant Graphics for Data Analysis. (Springer-Verlag, 2009). doi:10.1007/9780-387-98141-3.

48. R Core Team. R: A language and environment for statistical computing. R Foundation for Statistical Computing. (2013).

49. Conway, J. \& Gehlenborg, N. UpSetR: A More Scalable Alternative to Venn and Euler Diagrams for Visualizing Intersecting Sets. (2019). 
50. Baty, F. et al. A Toolbox for Nonlinear Regression in R: The Package nlstools. J. Stat. Softw. 66, 1-21 (2015).

51. Wickham, H. et al. Welcome to the Tidyverse. J. Open Source Softw. 4, 1686 (2019).

52. Auguie, B. \& Antonov, A. gridExtra: Miscellaneous Functions for 'Grid' Graphics. (2017).

53. Stamatakis, A. RAxML-VI-HPC: maximum likelihood-based phylogenetic analyses with thousands of taxa and mixed models. Bioinformatics 22, 2688-2690 (2006).

54. schrodinger/pymol-open-source. (Schrödinger, Inc., 2021).

55. Multiplexed RT-qPCR to screen for SARS-COV-2 B.1.1.7 variants: Preliminary results - SARS-CoV-2 coronavirus / nCoV-2019 Diagnostics and Vaccines. Virological https://virological.org/t/multiplexedrt-qpcr-to-screen-for-sars-cov-2-b-1-1-7-variants-preliminary-results/588 (2021).

56. Takada, K., Ueda, M. T., Watanabe, T. \& Nakagawa, S. Genomic diversity of SARS-CoV-2 can be accelerated by a mutation in the nsp14 gene. bioRxiv 2020.12.23.424231 (2020) doi:10.1101/2020.12.23.424231.

57. Variants: distribution of cases data. GOV.UK https://www.gov.uk/government/publications/covid-19variants-genomically-confirmed-case-numbers/variants-distribution-of-cases-data.

58. Preliminary genomic characterisation of an emergent SARS-CoV-2 lineage in the UK defined by a novel set of spike mutations - SARS-CoV-2 coronavirus / nCoV-2019 Genomic Epidemiology. Virological https://virological.org/t/preliminary-genomic-characterisation-of-an-emergent-sars-cov-2lineage-in-the-uk-defined-by-a-novel-set-of-spike-mutations/563 (2020).

\section{Figures}


PANGOLIN lineages, Russian samples
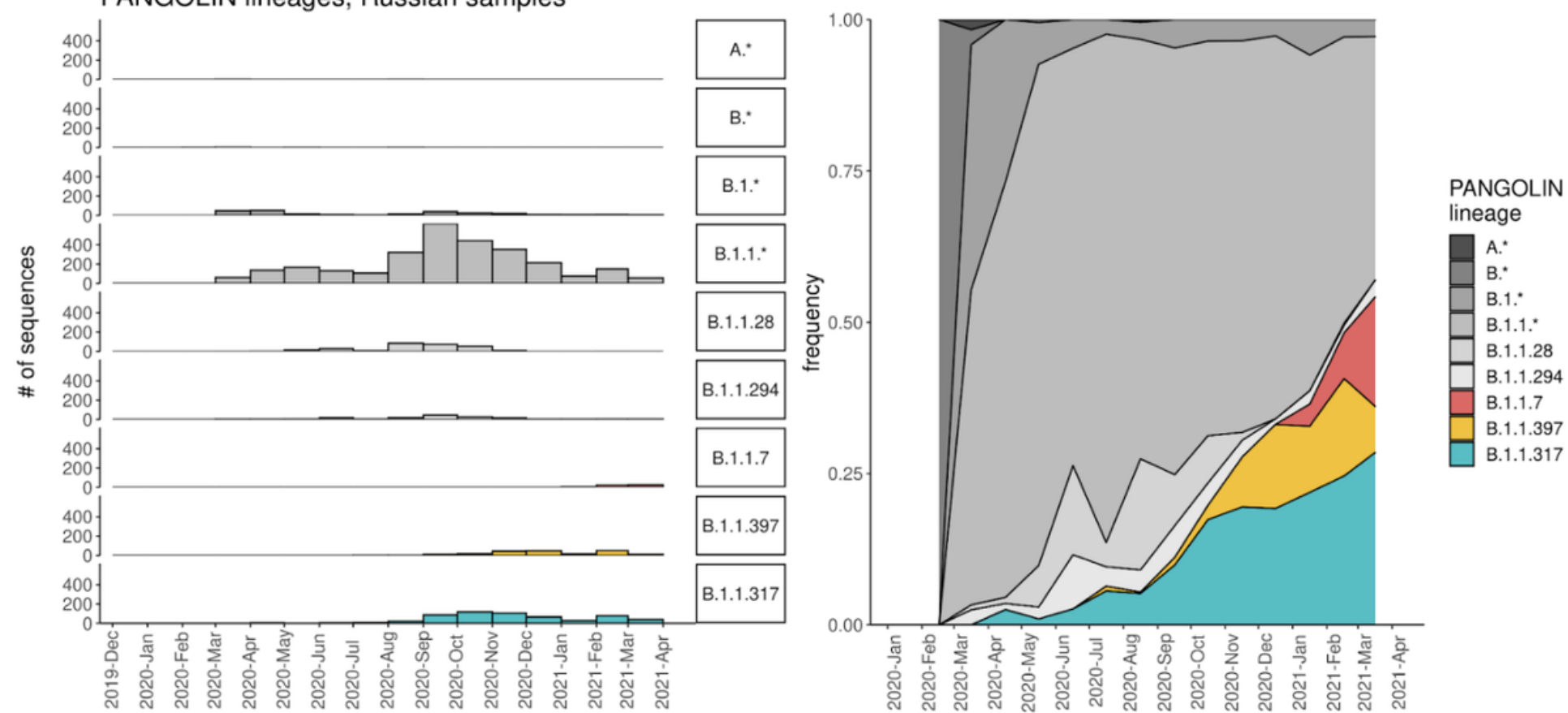

PANGOLIN lineages, non-Russian samples
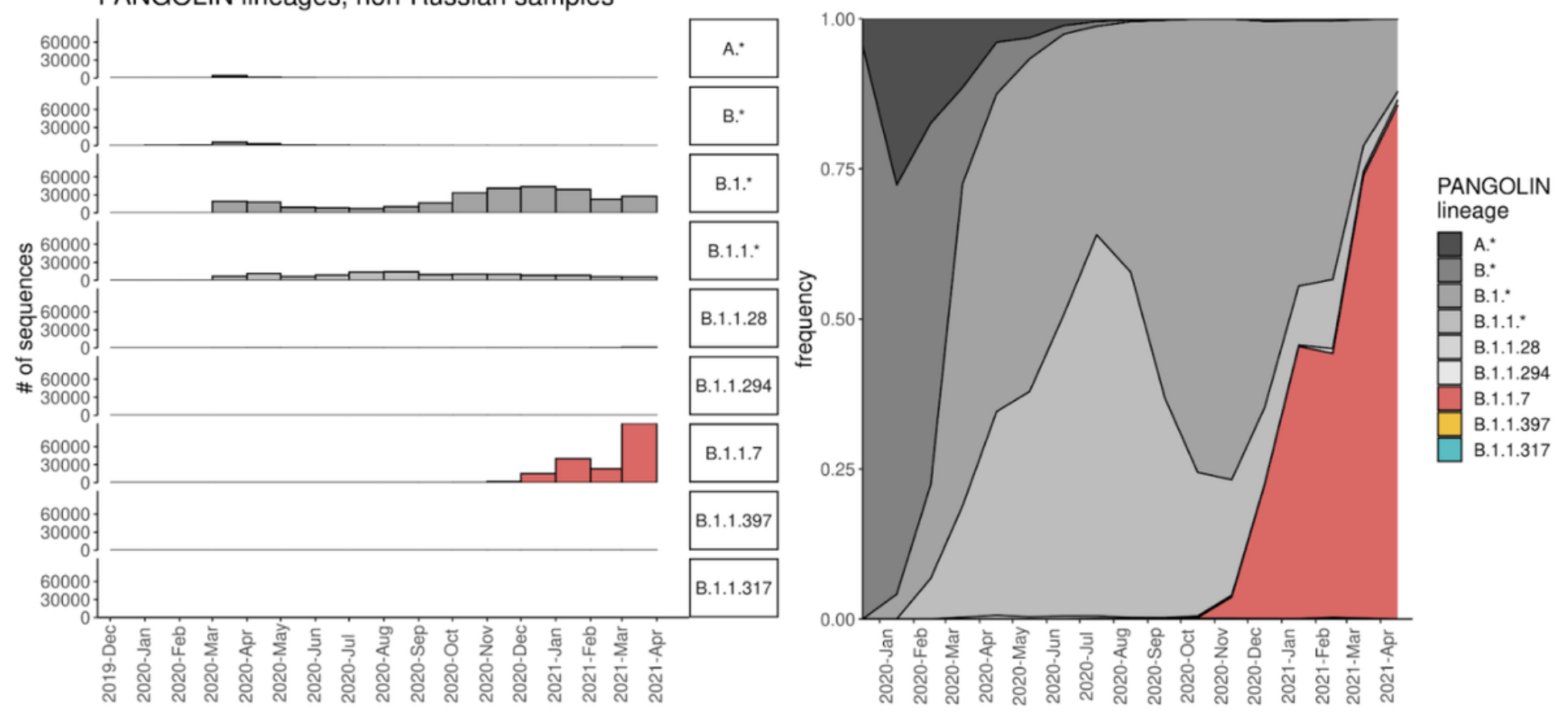

Figure 1

Dynamics of Pango lineage frequencies in Russia (top row) and among the non-Russian samples in GISAID (bottom row). Asterisks in Pango lineage designations correspond to pooled sets of lineages of that hierarchy level, except those listed in other categories; e.g., B.1.1.* includes B.1.1 and B.1.1.6 but not B.1.1.7 or B.1.1.317. Samples are split into one month bins. 

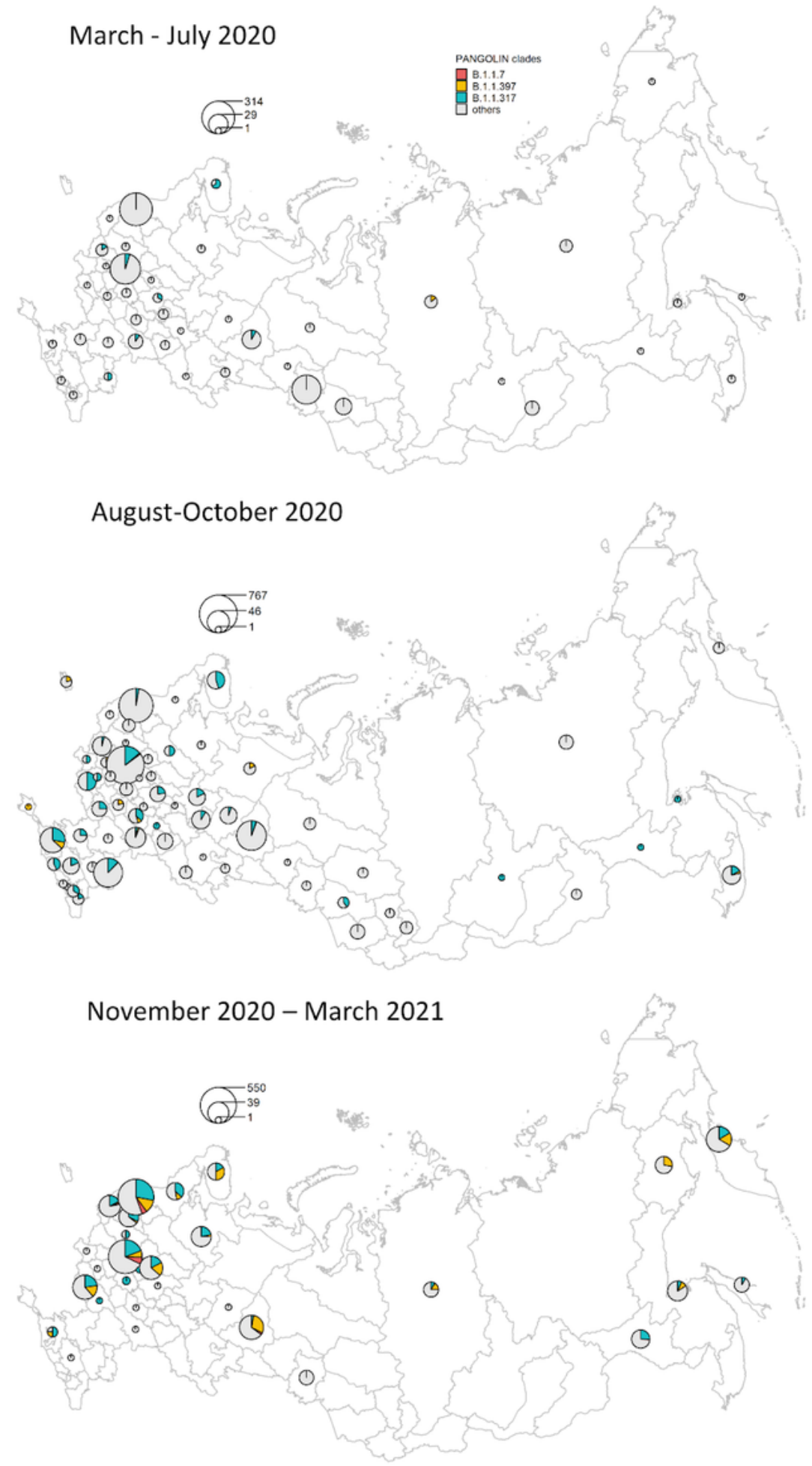

Figure 2

The spatio-temporal distribution of lineages that were frequent in Russia before April 2021. 

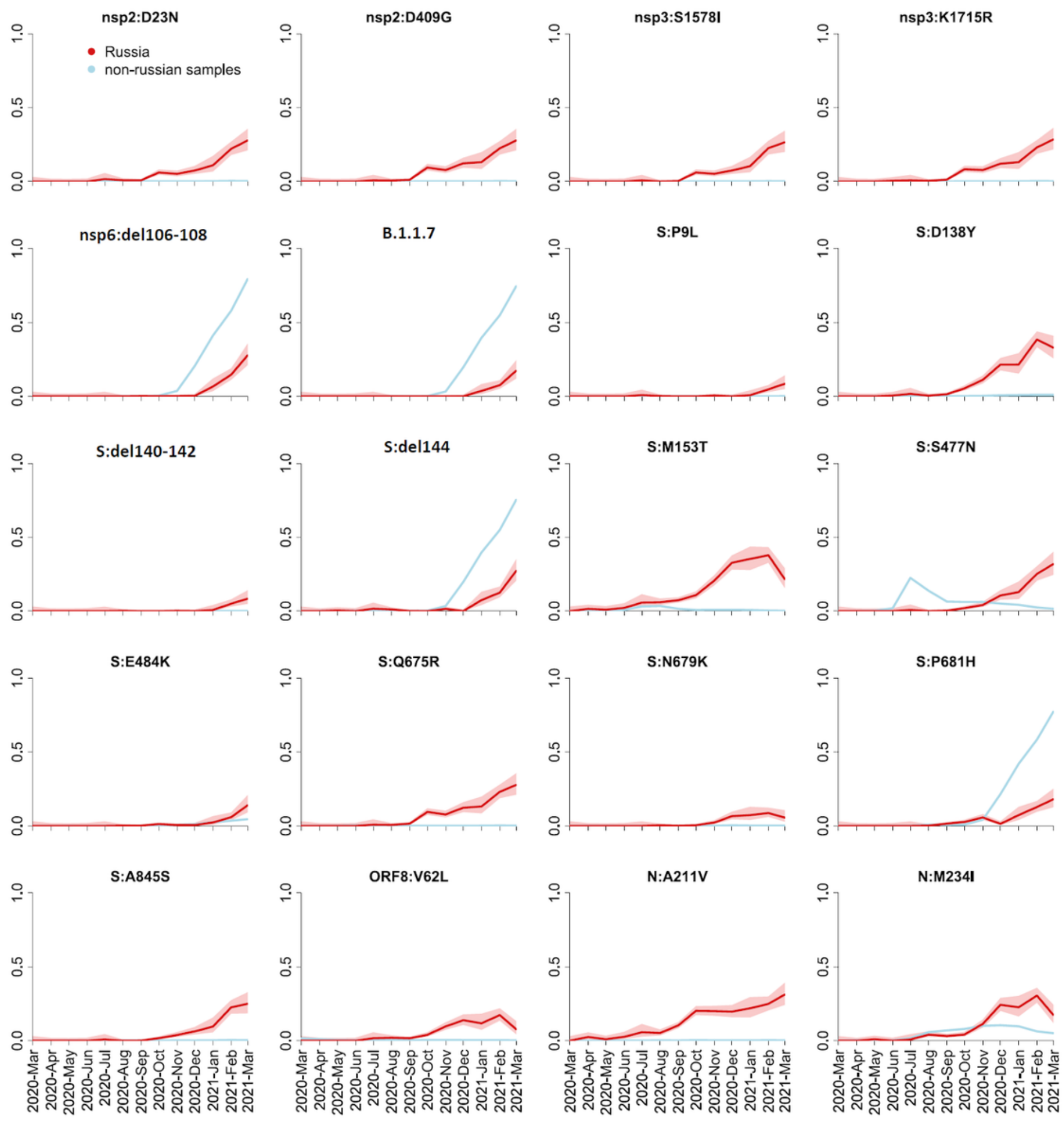

\section{Figure 3}

Frequency dynamics of SARS-CoV-2 amino acid changes. Plots represent changes in frequency over time for the non-reference amino acid mutations that reached frequencies above $10 \%$ ( $5 \%$ for the $S$ protein) among the 461 Russian samples obtained in February-March 2021. The frequency of B.1.1.7 is represented by the mutation nsp3:I1412T; deletions nsp6: $\Delta 106-108$ and $S: \Delta 144$ and substitution $\mathrm{S}: \mathrm{P} 681 \mathrm{H}$ which are a part of B.1.1.7 as well as other lineages are shown separately; the remaining 14 
mutations such that $>70 \%$ of samples carrying them belonged to B.1.1.7 are not shown. Changes in frequency in Russian (red) and non-Russian (blue) samples are shown in one-month time intervals. Shaded areas show $95 \%$ confidence intervals (Wilson score intervals).

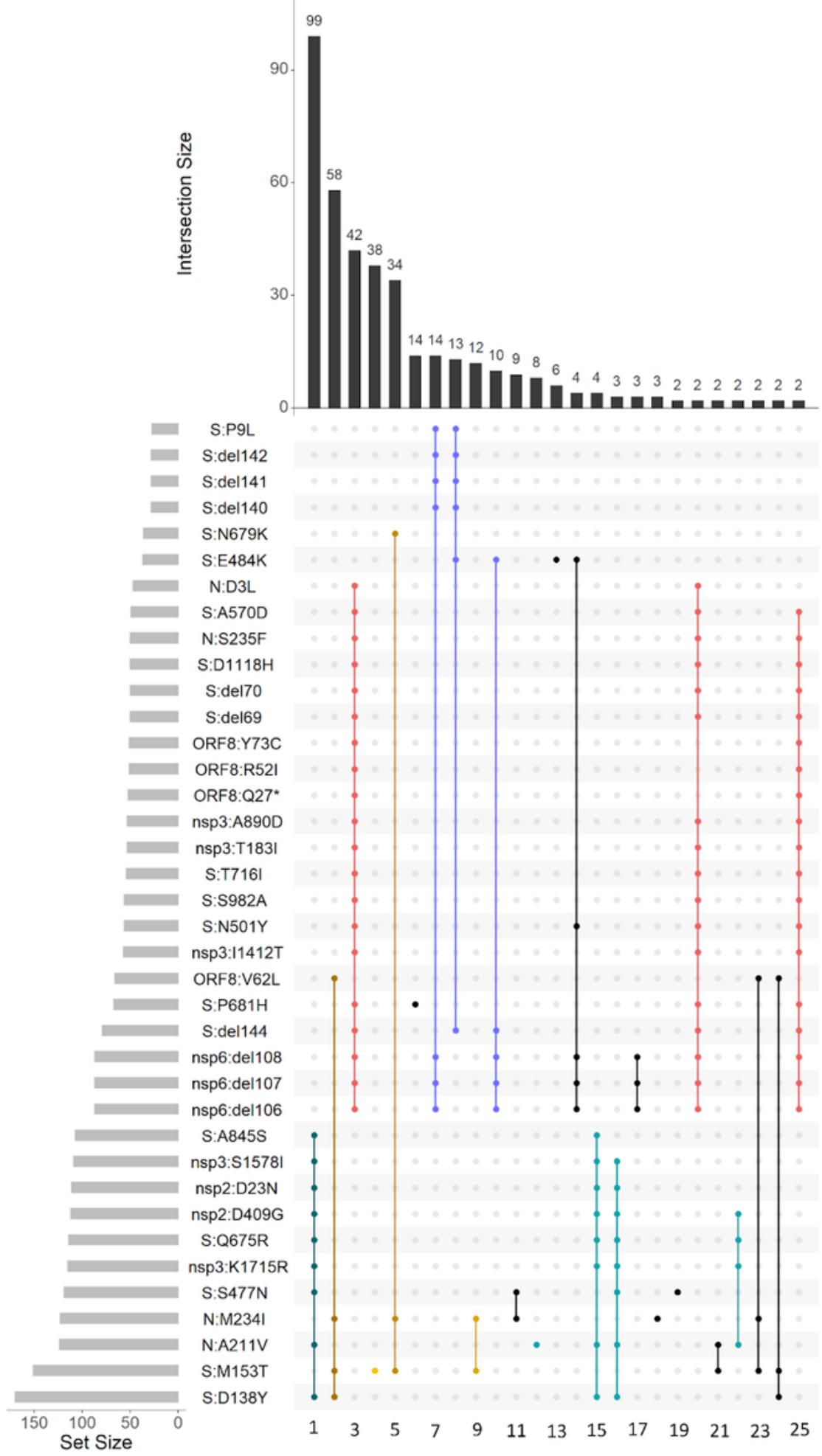

Figure 4

Variants with high frequencies in Russia in February-March. Horizontal rows represent all positions with non-reference alleles at frequency above 5\% (for spike protein) or 10\% (for other proteins) in Russia in 
February-March. Columns represent all observed combinations of these variants that included 2 or more samples, with black or colored dots indicating the presence of the non-reference variant. Colors dots represent the variants that are discussed in the text, with the same color coding as in Figs. 1, 2 and 5; blue color corresponds to variants B.1.1.524 (column 7), AT.1 (column 8) and B.1.1.525 (column 10).

a

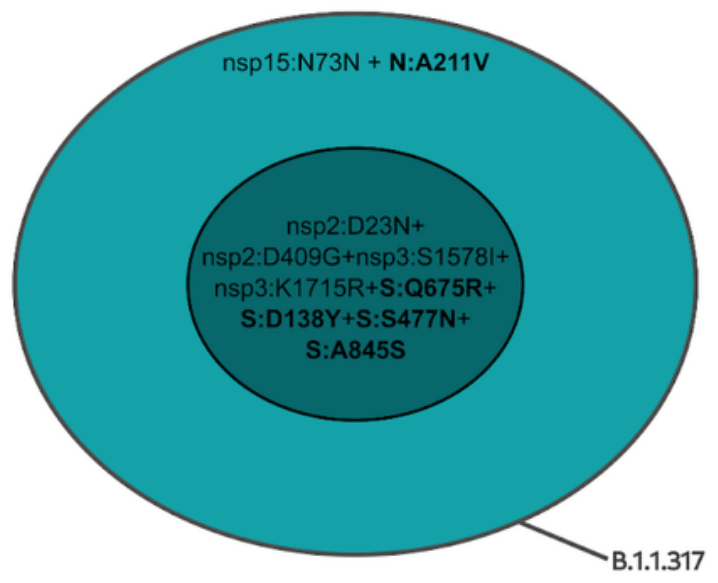

b

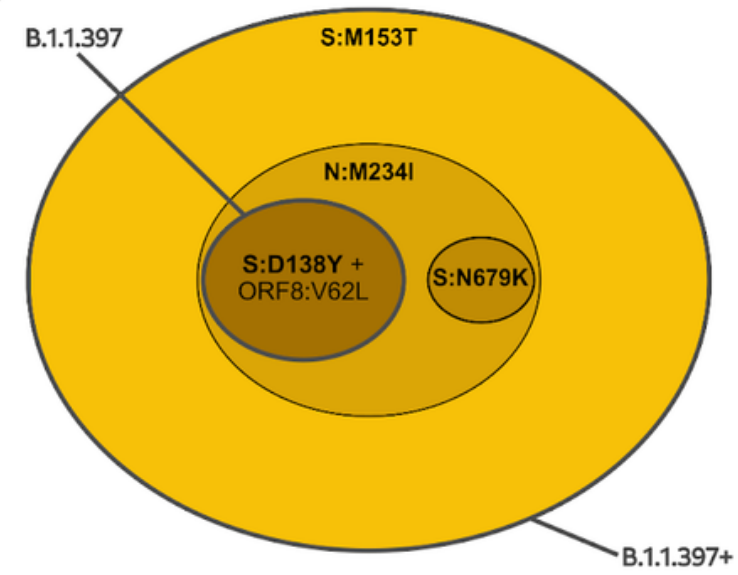

c

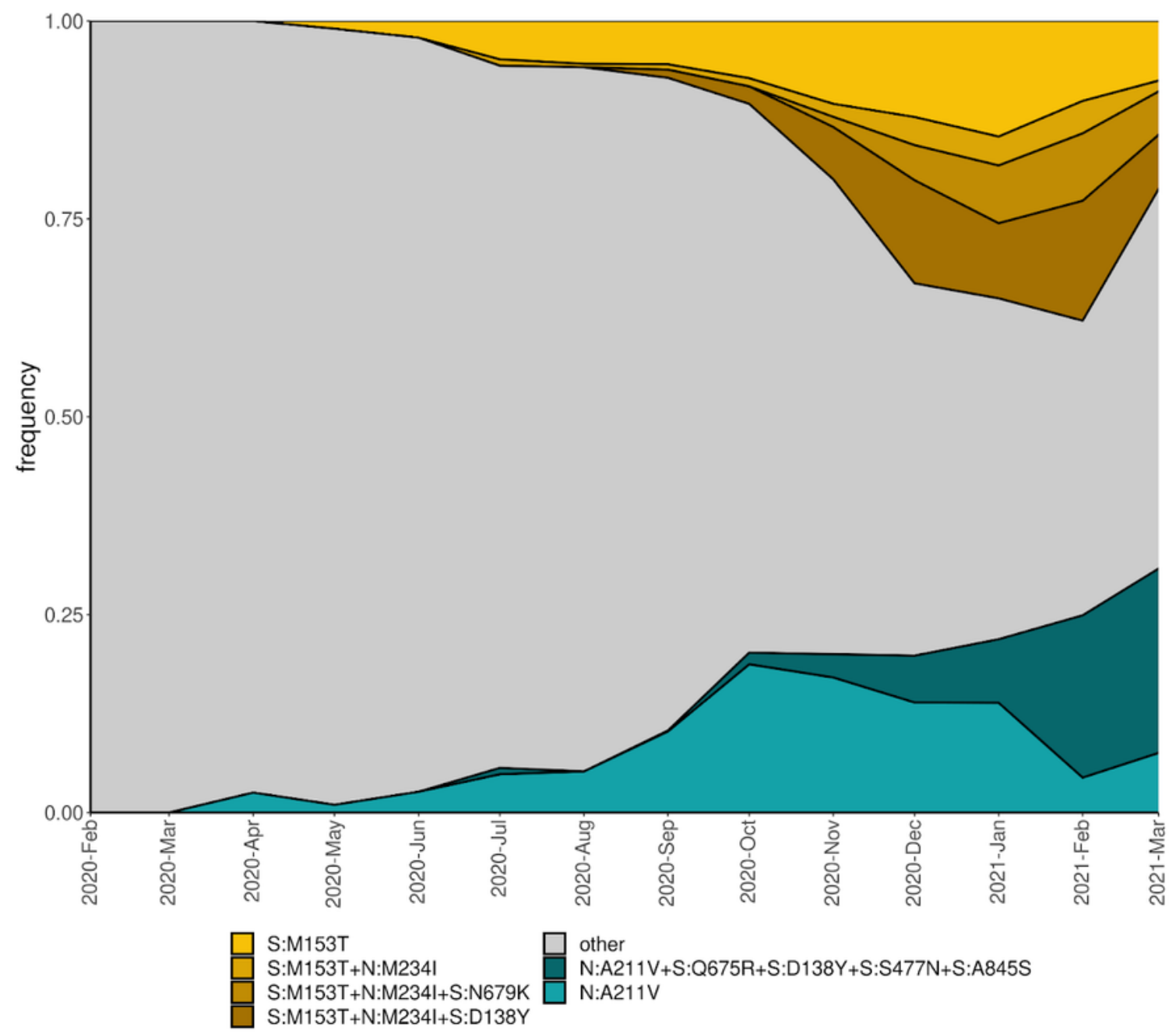

Figure 5 
Mutational composition and frequency dynamics of the B.1.1.317 and B.1.1.397+ lineages. A, B: schematic representation of the B.1.1.317 and B.1.1.397+ lineages. Pango lineage designations are approximate. C: Muller plots representing the frequency dynamics of the corresponding combinations of mutations in Russia.

A

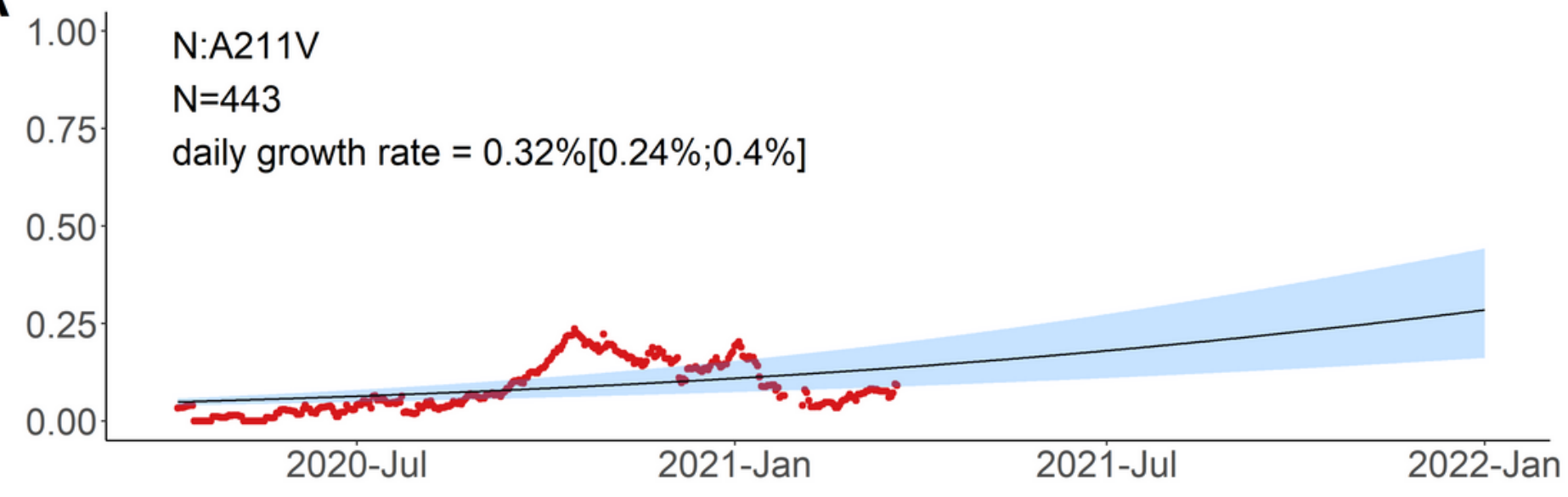

B

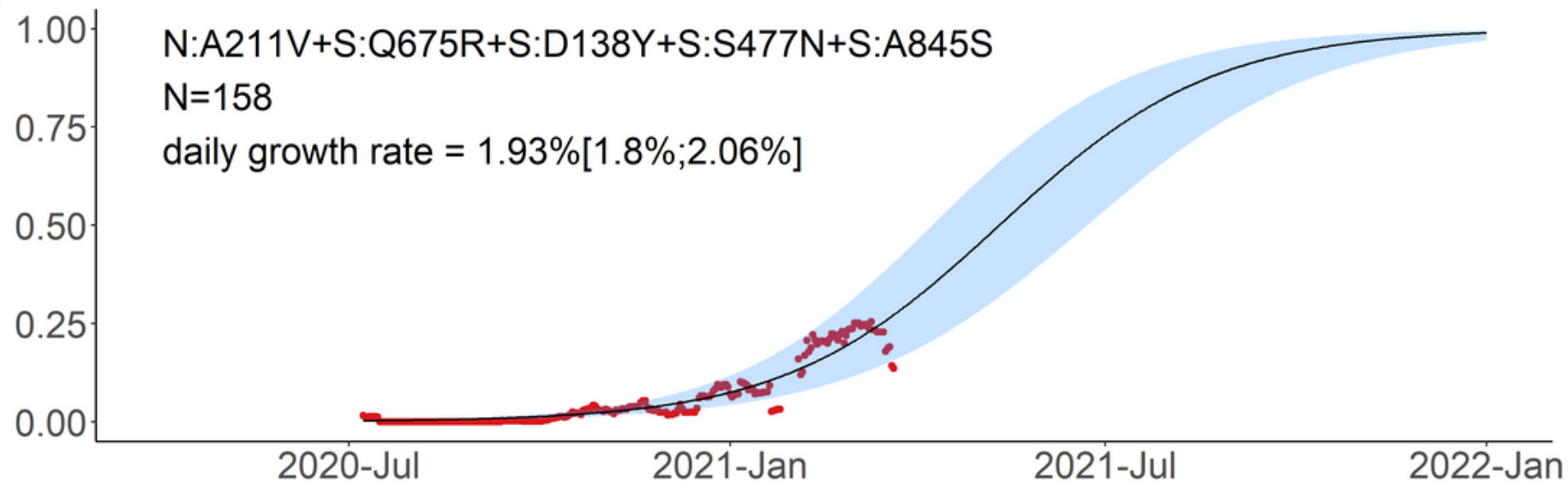

C

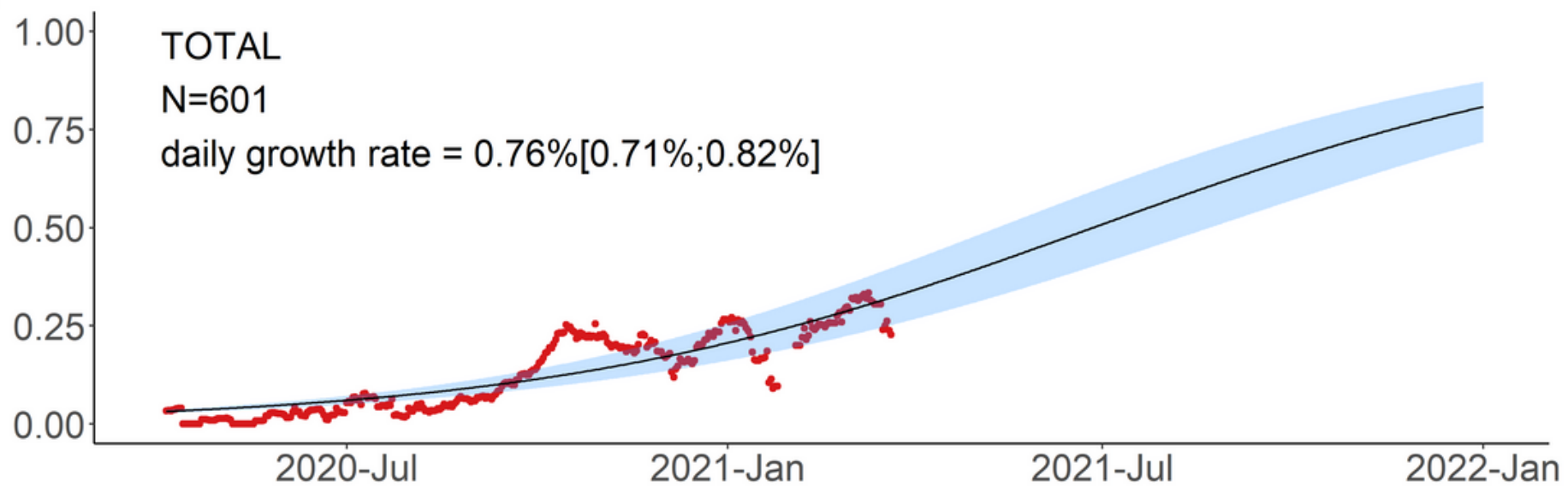

Figure 6 
Logistic growth model for nested variants defined by amino acid changes in the N:A211V context. Red dots, sliding window 14-day average frequency; shaded area, $95 \%$ confidence interval. Variants are identified according to the presence of the mutations in $\mathrm{S}$ and $\mathrm{N}$ proteins; see Fig. 5 for complete lists of mutations in the corresponding variants.

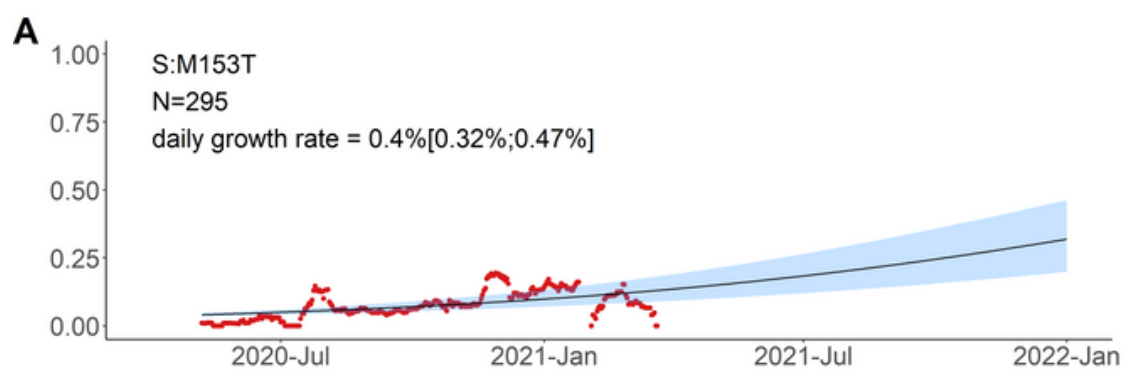

B
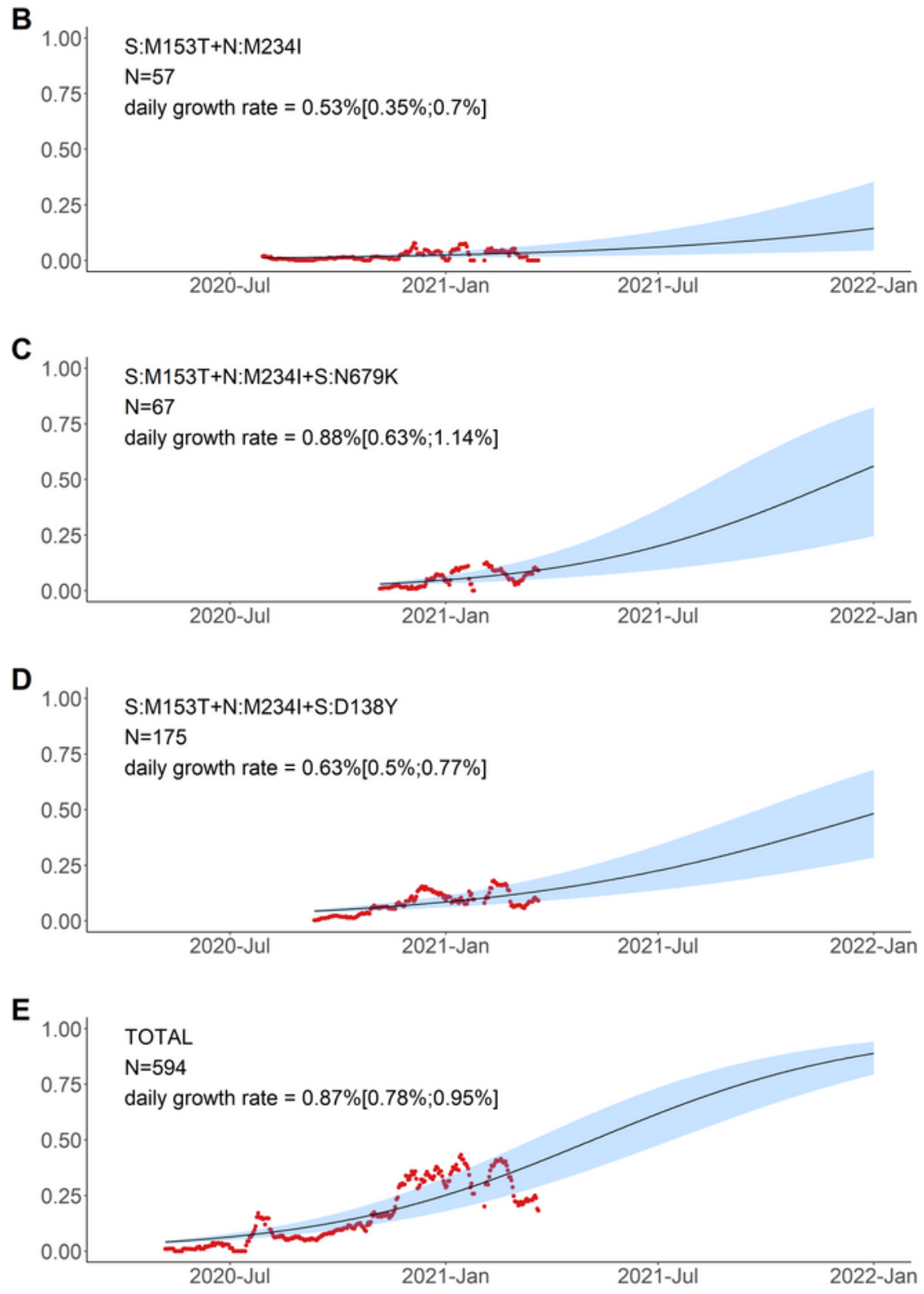

Figure 7 
Logistic growth model for variants defined by amino acid changes in the S:M153T context. Notations are the same as in Fig. 6.
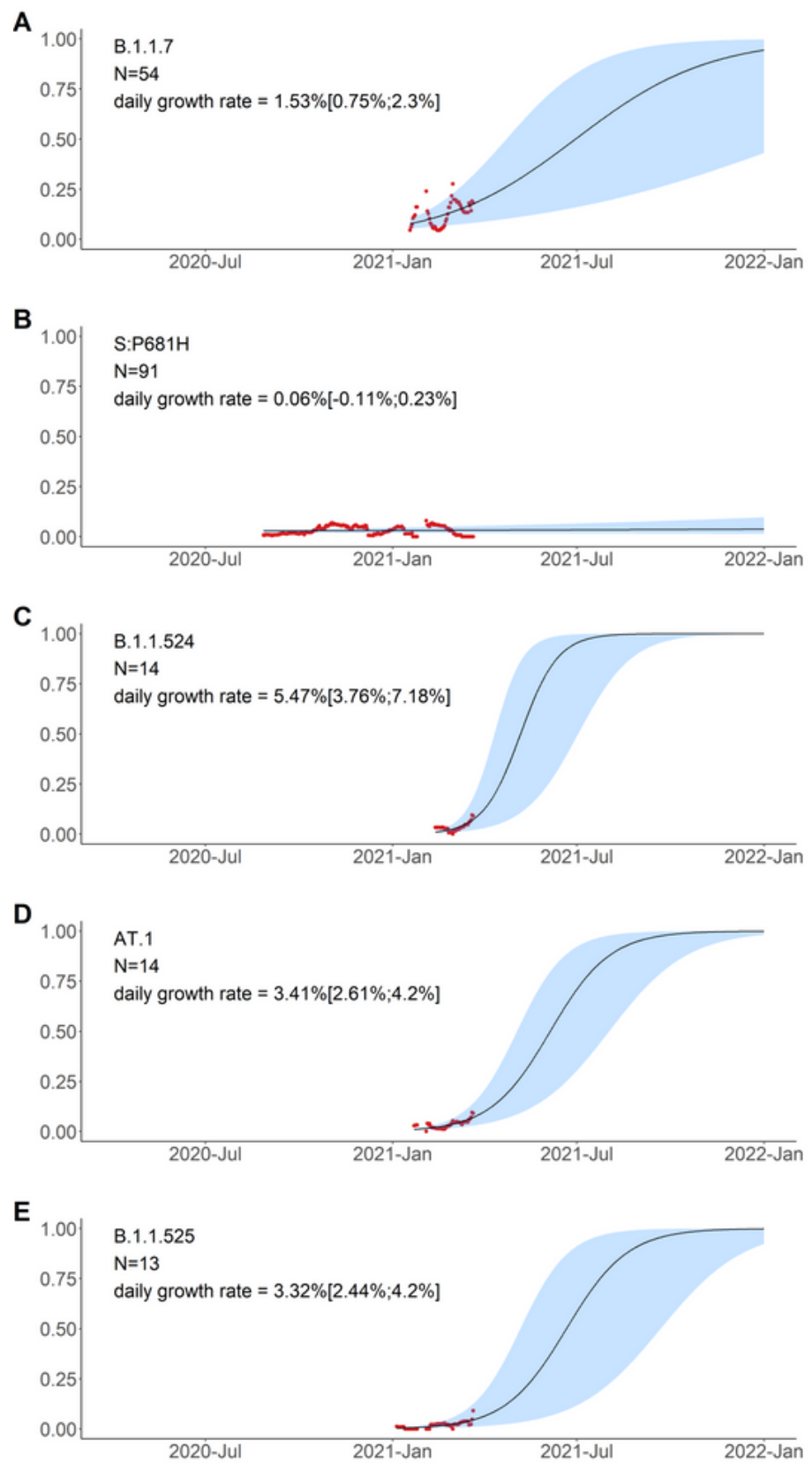

Figure 8

Logistic growth model for the five remaining amino acid variants with high frequencies in Russia in February-March. Notations are the same as in Fig. 6. S:P681H has been observed both independently and 
as part of B.1.1.7; in panel B, the cases of B.1.1.7 are not shown, by excluding the S:P681 H+nsp3:I1412T combination.
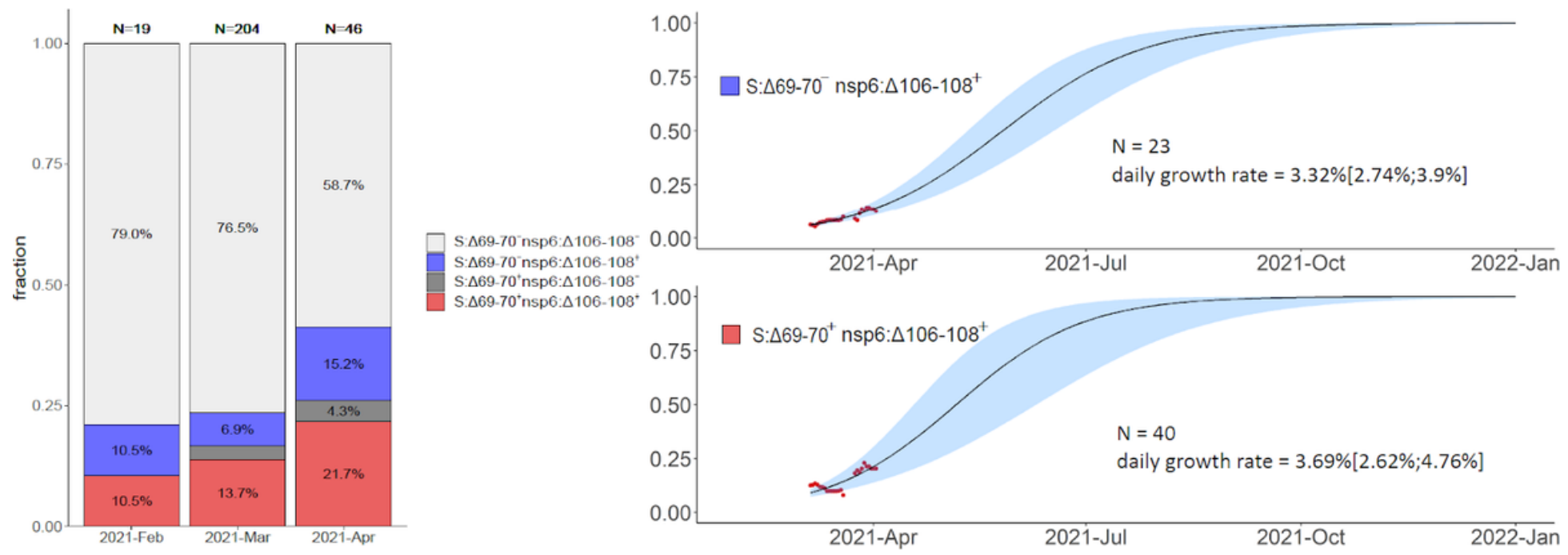

\section{Figure 9}

Frequencies of S: $\Delta 69-70, n s p 6: \Delta 106-108$, and their combination in Russia in Feb-Apr 2021 based on PCR data. S: $\Delta 69-70+$ nsp6: $\Delta 106-108+$ samples correspond to B.1.1.7, and S: $\Delta 69-70-n s p 6: \Delta 106-108+$ samples correspond to B.1.1.524 or B.1.1.525. The rare instances of S: $\Delta 69-70+n s p 6: \Delta 106-108$ - probably correspond to false positive S: $\Delta 69-70$ detection. Notations for logistic curves are the same as in Fig. 6 . 


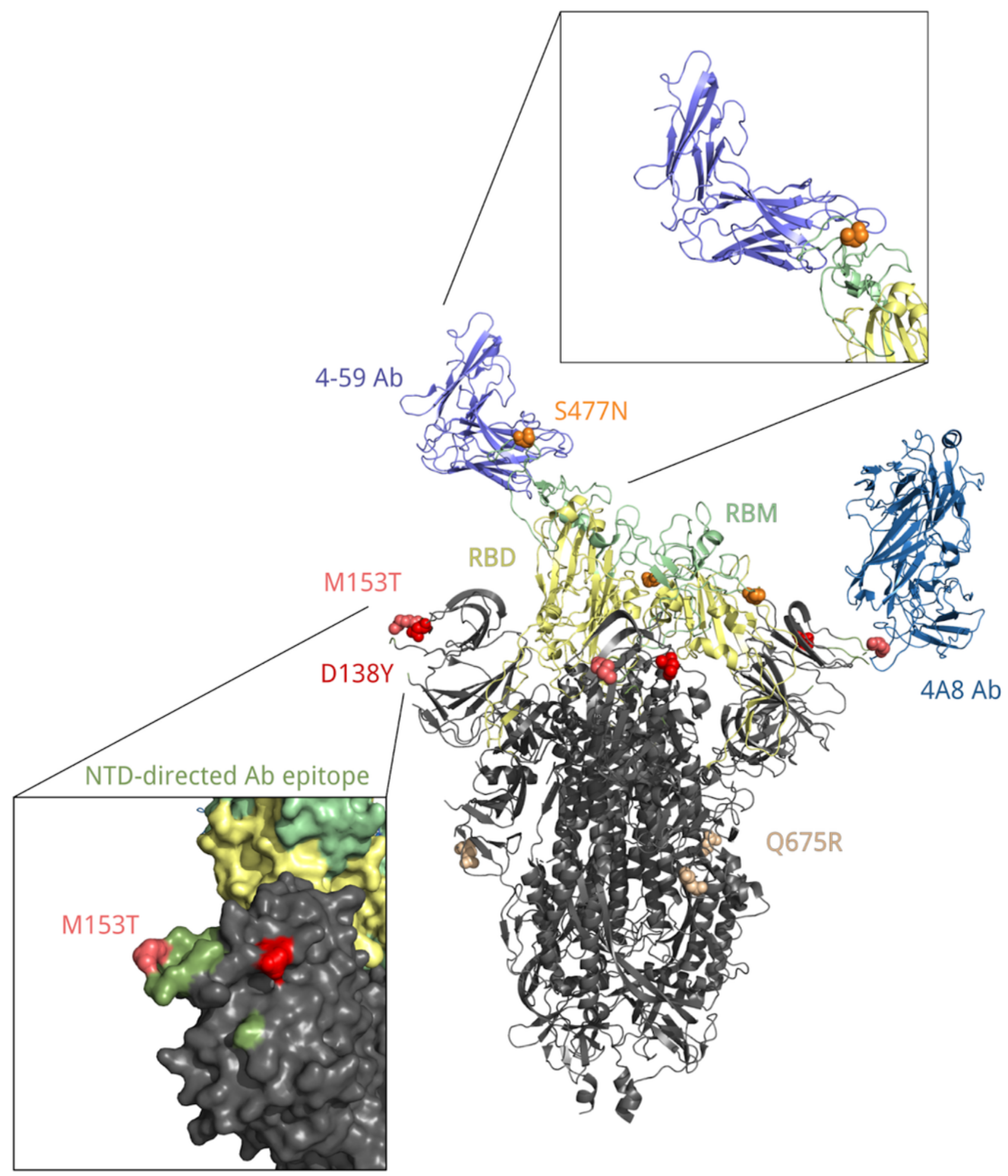

Figure 10

Position of residues 138, 153, 477 and 675 in the spatial structure of the S protein bound with 4A8 and 459 antibodies (PDB IDs: 7c2I and 7czx). Each of these residues is colored in its own color. Yellow, receptor binding domain; green, receptor binding motif; ocean blue, heavy chain of the 4A8 antibody; blue, heavy chain of the 4-59 antibody; olive, antibody binding epitope. 


\section{Supplementary Files}

This is a list of supplementary files associated with this preprint. Click to download.

- FigureS1.pdf

- SupplementaryFile1.pdf

- SupplementaryFile2.acknowledgmentsGISAID.zip

- Supplementary.pdf 